\title{
Effets premiers de l'intervention
}

Le cas de problèmes complexes (TMS - RPS) en santé au travail

First effect of the intervention. The case of complex problems (MSD - PSR)

in health at work

\section{Sandrine Nahon, Célia Queriaud, Cécile Mege Piney et Isabelle Thibault}

\section{(2) OpenEdition}

1 Journals

Édition électronique

URL : http://journals.openedition.org/activites/3582

DOI : 10.4000/activites.3582

ISSN : $1765-2723$

Éditeur

ARPACT - Association Recherches et Pratiques sur les ACTivités

Référence électronique

Sandrine Nahon, Célia Queriaud, Cécile Mege Piney et Isabelle Thibault, «Effets premiers de l'intervention », Activités [En ligne], 15-2 | 2018, mis en ligne le 15 octobre 2018, consulté le 01 mai 2019. URL : http://journals.openedition.org/activites/3582 ; DOI : 10.4000/activites.3582

Ce document a été généré automatiquement le 1 mai 2019.

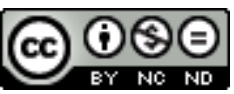

Activités est mis à disposition selon les termes de la licence Creative Commons Attribution - Pas d'Utilisation Commerciale - Pas de Modification 4.0 International. 


\title{
Effets premiers de l'intervention
}

\author{
Le cas de problèmes complexes (TMS - RPS) en santé au travail \\ First effect of the intervention. The case of complex problems (MSD - PSR) \\ in health at work
}

Sandrine Nahon, Célia Queriaud, Cécile Mege Piney et Isabelle Thibault

\section{NOTE DE L'ÉDITEUR}

Article soumis le 15 avril 2017, accepté le 30 août 2018

\section{Introduction}

1 Depuis de nombreuses années, la question de l'évaluation de l'intervention sur les Troubles Musculo squelettiques (TMS) et les Risques Psychosociaux (RPS) est une préoccupation pour les acteurs universitaires, institutionnels et praticiens. L'intérêt se porte à la fois sur la pertinence et la cohérence de l'intervention, et sur sa productivité, ses effets et le contexte d'implantation dans la production de ces effets (Avargues, Champagne, Contandriopoulos, \& Denis, 2000). En 2017, à partir d'un recensement d'interventions dites " complexes ", l'INRS et l'ANACT ont publié un guide à l'intention des préventeurs et des intervenants en santé au travail pour les aider à évaluer les interventions de prévention des TMS et RPS.

2 Nos constats issus d'une pratique en Cabinet Conseil depuis plus de 25 ans dans le domaine de la Santé (TMS, RPS, Handicap) sont unanimes. Quel que soit le type d'interventions réalisées, l'intervenant confronté à ces sujets complexes constate non seulement que l'issue est incertaine (progression des RPS \& TMS, difficultés du maintien en emploi pérenne), mais se questionne aussi sur les indicateurs capables de témoigner de la pertinence du dispositif ou de la stratégie d'intervention qu'il mobilise. En effet, face à la complexité et à la multi dimensionnalité des environnements d'interventions, l'intervenant est pris dans un tourbillon d'éléments, de données (mode de production, 
organisation du travail, relations entre acteurs, etc.), où il lui devient difficile de repérer les signaux, indicateurs des «effets » de sa propre intervention. Ceci est d'autant plus ardu, notamment pour les interventions dans les domaines des TMS et RPS, que les effets peuvent d'une part être multiples et différés avec des améliorations progressives, peu tangibles, voire invisibles; d'autre part, être plus ou moins masqués par les changements structurels et organisationnels que connaissent les organisations lors du déroulementmême de l'intervention.

Pourtant, savoir reconnaitre les signaux qui identifient les transformations apportées par l'intervention est un véritable enjeu pour l'amélioration durable des conditions de travail et de la performance des organisations (Daniellou, 2006).

La capitalisation des interventions menées au sein de notre Cabinet Conseil nous a permis d'identifier cinq signaux à détecter, précurseurs d'«effets premiers»: une nouvelle lecture partagée de l'existant, une nouvelle appréciation de la valeur, une évolution du périmètre de l'intervention vers les activités managériales et organisatrices du travail, un élargissement des catégories d'acteurs "embarqués », et, une construction de nouveaux standards et outils de gestion qui se distinguent par une intégration des critères de santé et de performance économique.

5 Nous émettons l'hypothèse que ces signaux, plus facilement discernables sur le temps de l'intervention que les effets sur la santé dont la mise en visibilité nécessite un temps long, ont une valeur prédictive. L'objectif de cet article est de caractériser ces «effets premiers », en illustrant nos propos par un exemple de dispositif d'intervention dans le secteur des transports.

\section{1. Évaluer une intervention, c'est compliqué !}

\section{1. Évaluer, mais quoi?}

6 Notre expérience terrain fait remonter des interrogations que l'on retrouve dans la littérature: évaluer nos interventions n'est pas simple et pour diverses raisons (Daniellou, 2006) :

- L'écriture de la proposition d'intervention confère un caractère d'auto-prescription

- Les objectifs se construisent au fur et à mesure avec la compréhension de la «théorie sousjacente ", quelque peu dépendante de l'intervenant aussi ;

- Des réajustements ont lieu en cours d'intervention;

- Une investigation est nécessaire, celle de la stratégie d'implantation avec les composantes formelles et les composantes réelles de l'intervention;

- L'expérience humaine et le vécu au-delà des étapes visibles de l'intervention ont un impact sur l'évaluation;

- La perception de la douleur physique et/ou psychique complexifie considérablement l'évaluation des effets sur la santé, notamment pour les interventions de prévention des TMS et/ou RPS ;

- Les indicateurs adaptés pour l'évaluation émergent presque naturellement plutôt à la fin de l'intervention;

- Il y a un risque de focalisation sur les indicateurs si ceux-ci sont affichés en amont ;

- La comparaison avant / après peut être biaisée : l'avant notre arrivée est difficilement palpable et l'évaluation après intervention peut être conditionnée par un changement d'acteurs ou une appropriation des sujets par les acteurs entreprise ; 
- Évaluer l'intervention, c'est aussi évaluer nos capacités de diagnostic, mais aussi à convaincre ;

- Évaluer nos difficultés implique de garder des traces, voire de constituer un carnet de bord, ce qui peut être coûteux.

7 Qui plus est, le praticien qu'est l'intervenant développe une forme d' «art » dans un univers « mouvant » (Schön, 1994), une pratique d'autant plus difficile à évaluer. En effet, selon l'auteur, la pratique professionnelle, ponctuée par la complexité, l'incertitude, l'instabilité, le particularisme et les conflits de valeurs des situations étudiées, permet de développer une certaine dimension «artistique» pour gérer les situations auxquelles l'intervenant doit faire face.

8 Tout comme les travaux sur la recherche évaluative d'Avargues, et al. (2000) qui pointent les éléments à évaluer dans les interventions, Landry (2008) propose d'investiguer la stratégie sous-jacente de l'intervention et la stratégie d'implantation à la mise en œuvre de l'intervention, tout au long de celle-ci. Toute évaluation doit se faire suivant le problème posé et le contexte dans lequel elle se déroule. Dans cette lignée, nous avons choisi un prisme plus restreint, mais plus accessible dans l'immédiateté de l'intervention : investiguer les effets produits par l'intervention, les mises en mouvements provoquées.

\subsection{Regarder les effets produits, mais dans quelle temporalité ?}

Le schéma général des relations entre travail et santé (Volkoff \& Molinié, 2011) au fil de l'existence illustre différents types d'effets de la santé sur le travail et du travail sur la santé. Cette approche diachronique nous permet d'axer notre regard sur l'activité de travail définie comme " médiatrice » dans les relations travail-santé et sur les régulations associées pour gérer les perturbations qui émergeraient des interactions entre l'homme au travail et le système de travail. Cette approche nous invite à appréhender l'activité présente de façon synchronique, celle d'ici et maintenant, avec une perspective diachronique, l'activité dans un temps long. Ainsi, nous reprenons à notre compte qu'évaluer correspond à regarder l'immédiateté à travers les effets émergeants dans nos interventions, tout en se projetant dans le temps de l'intervention, puis dans le temps audelà de l'intervention.

\subsection{Des effets à investiguer de quelle nature?}

Pour les interventions sur des problématiques aussi complexes que celles des TMS et des RPS, une de nos principales préoccupations est de savoir si nous sommes parvenus à «faire bouger des choses », tant au niveau des représentations et de la compréhension des situations de travail qu'au niveau du développement possible et conjoint des différents acteurs et des changements impulsés. En ergonomie constructive, l'intervention est structurée autour d'une question centrale (Arnoud \& Falzon, 2017) : comment favoriser le développement conjoint des individus, des collectifs et de l'organisation? Deux méthodes d'intervention, qui sont dans la réalité souvent conjuguées, se distinguent alors: celles qui visent le développement d'un référentiel partagé, et celles qui visent à construire un futur possible. Ces travaux nous guident pour caractériser nos effets à évaluer, nous confirment le regard à apporter sur la « mise en mouvement ». 
11 Pour Heddad et Biquand (2015), l'intervention doit pouvoir contribuer à promouvoir et développer de nouveaux processus de décision et de régulation en réintégrant le travailleur dans les cycles de décisions qui conditionnent son travail : "Intervenir c'est initier de nouvelles articulations structurelles et organisatrices pour de nouvelles marges de manouvre et pour faire exister le point de vue du travail ». Reste pour nous à vérifier si cette optique s'exprime dans notre évaluation des effets.

Enfin, nous souhaitons placer nos capteurs d'effets d'intervention à un niveau suffisamment élevé de l'organisation du travail de façon à pouvoir observer des changements visibles au sens de la macro-ergonomie, comme le propose Hendrick (2003). Selon ce dernier, ces changements à leur tour entraînent d'autres changements qui, petit à petit, se traduiront dans des indicateurs traditionnels de la santé au travail.

L'objet de notre propos n'est pas tant de mesurer notre efficacité, mais plutôt de saisir en temps réel, les effets en cours, leur objet, avec notre perception et notre compréhension des mouvements associés. Nous l'illustrerons par une intervention conséquente dans une grande entreprise de transport.

\section{L'exemple d'une intervention dans le secteur des transports}

\subsection{Un historique de discordances entre Direction et agents}

14 L'intervention support de nos propos s'est déroulée dans un des établissements d'une entreprise de transport. Elle avait la particularité de débuter consécutivement à des suicides et dans un contexte économique tendu. Les tensions sur les effectifs, les salaires et l'absentéisme étaient au cœur des discussions sur la dégradation physique et psychosociologique de la santé. C'est pourquoi la demande initiale d'intervention avait des attendus en termes «d'amélioration de la qualité de vie au travail au sein de l'établissement ".

15 Au démarrage de l'intervention, Direction et agents étaient dans une situation de rupture. Des incompréhensions et des dialogues de sourds s'étaient installés entre eux : « Nous vous parlons de nos soucis, vous nous parlez de vos priorités» (verbatim des organisations syndicales). Ces différences de points de vue exprimaient-elles une incompatibilité entre santé et performance ou étaient-elles deux facettes différentes du même problème?

Les difficultés vécues et exprimées par les agents semblaient liées à l'évolution de leur métier en termes de contenu des activités, de spécialisation, de gestes métiers, de parcours professionnel, et, aux conditions de réalisation du travail notamment pour faire face aux comportements agressifs de certains clients ou aux situations d'isolement. Par ailleurs, la Direction de l'établissement devait maintenir l'atteinte d'objectifs de production, dans un contexte social et économique délicat vis-à-vis des commanditaires et des Directions métiers centrales.

17 Cependant, c'est bien «sur les conditions de travail des agents » et "uniquement sur celles-ci » que Direction et partenaires sociaux décrivent le périmètre retenu du début d'intervention. 


\subsection{Des attentes fortes de co-construction des pistes de solutions}

Dans ce climat social tendu, les attentes de l'intervention ont été reformulées sur :

- Une approche dynamique et systémique (au-delà des collègues des agents directement concernés) pour mettre en évidence l'ensemble des facteurs qui permettent la compréhension de la situation vécue par les agents, et pour comprendre l'articulation de ces facteurs ;

- Une approche participative pour mobiliser l'ensemble des parties prenantes pertinentes dans l'élaboration du diagnostic et la recherche de solutions ;

- Une approche de l'activité pour reconnaître les situations réelles de travail, favoriser l'expression des difficultés vécues au travail ;

- Une démarche orientée vers l'action pour produire des résultats concrets dans la vie au travail des agents et des managers. L'intervention avait comme objectif de contribuer à restaurer la confiance entre les acteurs de terrain, notamment Direction et Organisations Syndicales. Pour cela, il convenait de veiller à créer les conditions de développer un langage commun, à partager les éléments de diagnostic et à co-construire les pistes de solutions.

\subsection{Partis pris de l'intervention}

Le dispositif méthodologique mis en place lors de cette intervention a reposé sur l'analyse croisée des conditions de travail et des relations qu'entretenaient trois métiers centraux pour la production du service (agents de contrôle, organisateurs de l'activité, managers de proximité), et plus largement les services supports, la Direction de l'établissement et des activités. Ces trois métiers avaient été ciblés suite à la tenue de deux CHSCT extraordinaires. Pour répondre aux attentes de l'entreprise, notre démarche a été structurée en assurant :

- Le paritarisme (comité de pilotage paritaire);

- La participation (réalisée avec les salariés);

- La concertation (validations successives tout au long de sa progression), avec notamment des phases de pré-restitution.

Le calendrier de l'intervention prévoyait la réalisation d'un diagnostic partagé à 3 mois et un plan d'actions opérationnel à 6 mois. Les moyens d'investigation mis en œuvre (entretiens, groupes de travail métiers et inter métiers, observations) ont permis de développer un point de vue partagé de la situation, et ce, pour les trois métiers. Pour nos interlocuteurs, les temps d'observation terrain des agents (associés à l'approche ergonomique) ont été également convenus afin de tisser des liens entre le vécu et les faits, l'objectivité et la subjectivité, en bref resynchroniser « le son et l'image».

Ce dispositif a été animé par une équipe pluridisciplinaire composée de quatre intervenants issus de disciplines différentes telles que l'ergonomie, les sciences de gestion, la sociologie, la psychodynamique du travail et la psychologie.

La participation active et pérenne de l'ANACT au sein de l'entreprise a fortement appuyé notre démarche, notamment pour maitriser le dialogue social. Ainsi, des éléments organisationnels à investiguer sont apparus dès le premier comité de pilotage. 


\section{Méthodologie}

Notre méthodologie de recherche se compose de trois phases:

1. La réalisation d'une intervention avec un dispositif d'évaluation en continu ;

2. L'animation de notre dispositif de questionnement de la pratique et de mise en réflexivité collective au sein du cabinet avec des consultants hors intervention ;

3. L'analyse rétrospective de l'intervention.

Lors de la phase 1 de réalisation de l'intervention, le rebouclage dynamique et continu à chacune des étapes de l'intervention (cf. Figure 1) porte sur les effets produits immédiats ou non, les hypothèses d'effets au fur et à mesure des sujets et actions engagés par chacun des consultants et sur des partages de constats. Ce rebouclage a pour but de fournir des hypothèses d'actions, des orientations et des stratégies d'interventions ajustées. Cette première phase confère ainsi une évaluation en continu par l'équipe de l'intervention sur le court, moyen et long terme.

Figure 1 : Les quatre étapes de l'intervention. Figure 1: The four steps of the intervention

\section{4 ÉTAPES}

DONT L'ENCHAINEMENT SERA SOUS LA MATRISE ET LE PILOTAGE DU COPIL

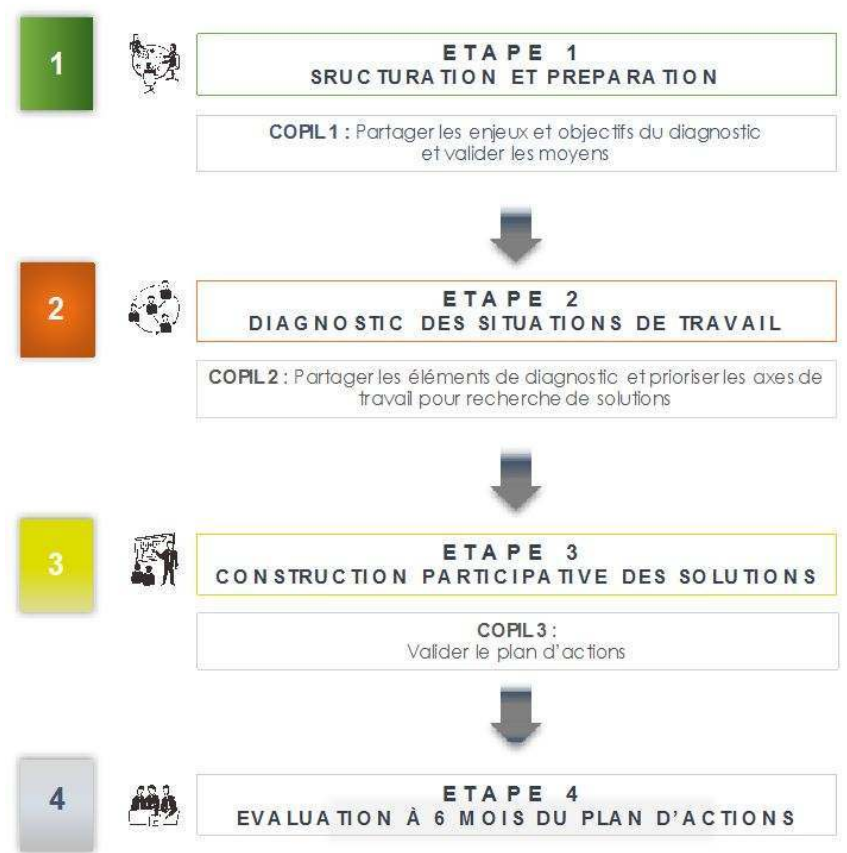

À la demande du terrain, l'intervention initiale de 49 jours a été complétée par 42 jours supplémentaires (cf. Tableau 1). Outre l'accroissement des temps de recueil de données par entretiens et groupes de travail, les comités de pilotage ont augmenté en nombre et en durée. Par exemple, le COPIL de restitution du diagnostic au comité paritaire est passé de 2 heures initialement prévues à 2 jours. Ce temps a été nécessaire pour aboutir à un document partagé du diagnostic où tous les mots ont pu être débattus avec l'ensemble des acteurs présents. 
Tableau 1 : Répartition prévisionnelle et effective des outils mobilisés lors de l'intervention. Table 1: Projected and effective distribution of mobilized tools during the intervention

\begin{tabular}{|l|c|c|}
\hline & Prévu & Réalisé \\
\hline Observations & 2 jours & 2 jours \\
\hline Entretiens COPIL & 12 entretiens & 18 entretiens \\
\hline Entretiens agents & 18 entretiens & 33 entretiens \\
\hline Entretiens fonctionnels & & 6 entretiens \\
\hline Groupe agents & 4 à 10 agents & 10 agents \\
\hline Groupe Responsables d'équipe & 4 à 10 agents & 8 agents \\
\hline Groupe organisateurs & 4 à 10 agents & 4 agents \\
\hline Groupe inter métiers & 4 à 10 agents & 7 agents \\
\hline CoPIL & 3 & 5 \\
\hline
\end{tabular}

La phase 2 de questionnement de la pratique et de mise en réflexivité avec l'ensemble des consultants du cabinet, participant ou non à l'intervention, permet d'identifier collectivement les points sensibles sur la détermination des effets, sur les produits de l'intervention et la valorisation de ces effets. Cette deuxième phase se déroule simultanément à la phase 1 , soit pendant la réalisation de l'intervention.

Enfin, la phase 3 d'analyse rétrospective, un an après la fin de l'intervention, permet d'en questionner le déroulement à l'aide de cinq entretiens semi-directifs. Ces entretiens, d'une durée variante entre 1 et 4 heures, ont été menés par une chargée R\&D n'ayant pas participé à l'intervention. L'analyse a été construite à partir d'une grille relevant six questionnements :

- Qu'a produit l'intervention ? À quel niveau ? À quel moment?

- Quelles actions ont pu être envisagées ? Pour quelles réactions?

- Quelle est l'originalité de cette intervention?

- Quel est votre vécu de l'intervention?

- Avez-vous des exemples significatifs?

- Avez-vous des éléments marquants lors de l'intervention?

Cette dernière phase a été engagée à la suite d'un travail réflexif sur l'évaluation auquel l'équipe d'intervenants a participé dans le cadre d'un groupe de travail pour l'élaboration du guide INRS et ANACT (2017).

L'utilisation de ces trois méthodes conjugue subjectivité et objectivité. La subjectivité relève des consultants qui peuvent apprécier au mieux les éléments contextuels tout au long de l'intervention. Pour ne pas limiter ce dispositif à une auto-évaluation et obtenir l'objectivité de l'évaluation, nous avons mis en place un dispositif collectif d'analyses : les 
revues de projets assurent un contrôle par une instance extérieure à l'intervention (Pagnac \& Arnaud, 2002), ce qui permet :

- D'amorcer les prises de conscience sur l'état de notre engagement;

- De passer d'un état de « pression individuelle » à un état de « décompression collective »;

- D'affiner la pédagogie de la communication autour de l'intervention pour faire progresser l'entreprise dans la recherche de solutions.

Notre dispositif méthodologique teste ainsi deux positionnements: l'évaluation en continue par l'équipe d'intervention sur le long terme, et, l'évaluation par l'analyse des effets. Les éléments pluridisciplinaires de l'intervention rendent son évaluation compliquée. C'est pourquoi nous avons choisi de ne pas l'évaluer directement, mais plutôt d'en comprendre les premiers effets.

\section{Effets premiers de l'intervention : du fonctionnement des organisations vers la construction de la santé au travail}

31 Cette intervention dans le secteur des transports va nous servir à caractériser les effets produits. Il est alors envisageable de distinguer deux types d'effets :

- Dans un premier temps, les effets premiers, c'est-à-dire des signaux indicateurs de la façon dont évolue le fonctionnement de l'organisation, sont visibles immédiatement et tout au long l'intervention. Ils sont donc facilement identifiables dans le nouveau fonctionnement ;

- Dans un second temps, les effets seconds apparaissent dans un temps plus long, notamment lorsqu'il s'agit d'impacts sur la santé des agents. D'ailleurs, leur nature hypothétique les rend difficilement visibles et évaluables.

Les effets premiers sont plus faciles à repérer dans le déroulement de l'intervention que les effets seconds. Nous proposons une identification à partir de cinq phénomènes prédominants, que nous illustrons au travers de l'exemple de notre intervention présentée ci-dessus.

\subsection{Identification des cinq signaux d'effets premiers de l'intervention}

\subsubsection{Une nouvelle lecture partagée de l'existant et/ou du passé}

La façon de regarder l'avenir est complètement dépendante de la façon de regarder le passé. Prenons l'exemple de la tenue des comités de pilotage lors de l'intervention. Dès les prises de rendez-vous pour fixer le premier comité de pilotage et lors de la tenue de celuici, les échanges ont permis d'identifier un décalage, qui en l'état devenait un point de rupture entre Direction et agents. Initialement, la demande adressée était ciblée sur un type de métier (niveau d'agents contrôleurs). Or, l'historique de l'établissement tout comme son actualité ont révélé des difficultés au niveau de la hiérarchie avec une équipe de Direction «mise à mal » et des responsables d'équipes « dépassés ». L'attention portée à la structure de l'activité managériale et à la qualité des articulations de l'organisation a permis de convenir que les managers partageaient tout autant les mêmes symptômes psychosociaux d'altération de la santé que les agents contrôleurs. Il en a été de même pour les organisateurs. 
L'intervention permet de revenir sur l'existant afin de travailler autour des différences de représentations selon les acteurs et leur positionnement dans l'établissement. La cible de l'intervention, ainsi élargie, a offert une tout autre perspective : il ne s'agit plus, pour chacun d'eux, de trouver des responsables, mais plutôt de (re)trouver un intérêt à travailler ensemble, à trouver des solutions collectivement. En constatant que ce mal était partagé, leur lecture de la situation s'en est trouvée dépassionnée par rapport à leur lecture précédente, qui menait à cette situation de rupture. Tous ont alors perçu une façon de regarder différemment le chemin futur: «je n'avais pas vu les choses comme ça ». Cette nouvelle lecture partagée a permis d'aboutir à un diagnostic commun, qui a pu être entièrement discuté par l'ensemble des parties prenantes. Ce diagnostic peut aujourd'hui être considéré comme un point de départ de reconstruction d'un langage commun inter-métier de manière à consolider les liens fragiles, et jusqu'alors dégradés, entre les différents mondes que sont les trois métiers étudiés.

\subsubsection{Une nouvelle appréciation de la valeur}

Le travail "non visible» par l'organisation ne donne en général pas lieu à sa quantification, ni à sa prescription, et encore moins à son évaluation. Pourtant, ce travail caché aux yeux de l'organisation est souvent ce qui permet d'atteindre les objectifs fixés et la qualité du service attendu. Dans le cas de notre intervention, pouvoir disposer d'un "petit temps » supplémentaire pour préparer le service est une véritable marge de manœuvre pour les agents, qu'ils utilisent pour réguler les différents aléas constitutifs au service produit, en limitant ainsi leur état de fatigue, voire de stress.

La prise en compte de l'appréciation de la valeur de ces nouvelles données, jusqu'alors invisibles, constitue un chaînon manquant à l'explication de la performance, à la construction de la reconnaissance du travail, et à l'articulation des relations entre les trois métiers. Ainsi, la mise en visibilité du caractère déterminant des phases de préparation de l'activité des agents, mises à mal par les évolutions récentes des équipements de travail et par l'organisation du travail, a permis de décaler le regard sur la valeur du travail de la préparation, ainsi que sur les moyens nécessaires pour mener à bien cette tâche.

\subsubsection{Une évolution du périmètre de l'intervention}

L'ouverture du champ de l'intervention vers les sphères de l'organisation, de la conception de ses règles, et $\mathrm{du}$ management des équipes marque la modification $\mathrm{du}$ périmètre de l'intervention, où vont se jouer les transformations, tant au niveau technique, structurel et organisationnel. C'est en effet un travail d'organisation du travail (Clot, 2002) auquel se sont livrés les acteurs dans les groupes de travail inter-métiers qui s'est rapidement substitué à la seule appréciation du vécu et du mal-être. Ainsi agents, organisateurs et managers ont travaillé à reconcevoir des règles de fonctionnement et d'organisation. Par exemple, des plages de 2 heures ont été prévues et testées dans les roulements pour assurer des entretiens entre agents et managers qui ne pouvaient se croiser que très rarement auparavant, compte tenu de leurs horaires et lieux de travail complètement décalés. Les 50 premiers entretiens selon cette nouvelle modalité organisationnelle ont été jugés très positifs: faire connaissance lors de première rencontre, évaluer sur les gestes métiers, débriefer sur les particularités et les incidents. 


\subsubsection{Un élargissement des catégories d'acteurs « embarqués »}

L'évolution du périmètre de l'intervention a amené « naturellement » les décisionnaires à s'inviter dans l'instance de pilotage paritaire. Ainsi, des représentants de la Direction nationale, qui ont le pouvoir d'agir sur les déterminants, ont embarqué dans le dispositif de travail: ils sont devenus acteurs en participant aux débats et en apportant des éclairages sur des orientations politiques et stratégiques. Ils ont ainsi offert à la fois la crédibilité nécessaire et les perspectives de changement. Il est probable que ces responsables se sont invités dans les réunions du comité de pilotage, car ils y ont vu un intérêt pour s'instruire, pour se nourrir de la qualité des données disponibles tant sur les problèmes que sur les solutions. À une plus large échelle, c'est également pour autoriser des transformations locales pouvant donner lieu à des expérimentations pilotes pour l'ensemble de l'organisation de l'entreprise.

\subsubsection{Une co-construction de nouveaux standards et outils de gestion}

Ces nouveaux standards et outils de gestion se substituent aux anciens en intégrant à la fois des critères de santé et de performance économique. Ces outils sont en général constitués à partir d'expérimentations menées sur des situations existantes ou simulées, à un niveau local. Le travail et les échanges sur celui-ci alimentent et enrichissent des outils de gestion, conçus à des niveaux décisionnels éloignés, de fait, de cette réalité. Par exemple, certains enchaînements entre destinations et horaires spécifiques ont été mis en évidence comme pouvant altérer la qualité de service et la qualité de vie au travail et hors travail. Notre dispositif d'intervention et l'instruction de pistes de solutions dans un groupe constitué de représentants des différents métiers ont permis de mettre en évidence l'utilité de s'appuyer sur une quantification de ces enchaînements comme un indicateur global de la performance. Ce dispositif de travail basé sur l'expérimentation demande cependant quelques prérequis :

- La possibilité d'itération en revenant plusieurs fois sur un sujet;

- Un espace préservé, presqu'invisible, accordé par ces tests en salle ;

- La réalisation de simulations-projections « réalistes »;

- La mise en évidence des avancées réalisées pas à pas, avec chaque pas consenti par chacun des métiers.

\subsection{Des effets premiers, précurseurs d'effets seconds}

L'idée d'ancrer l'évaluation sur ce qui évolue dans l'organisation n'est pas étrangère à la difficulté d'entrevoir d'emblée les effets seconds, notamment les effets sur la santé. Si des incidences sur la santé prennent leurs sources dans l'existant de l'organisation, il est normal que des modifications de l'organisation puissent à leur tour entraîner des conséquences sur la santé (cf. Figure 2). Les problèmes posés par l'évaluation sont alors multiples, car on ne sait pas, par définition, ni où, ni quand, ni comment enregistrer les effets sur la santé.

41 En effet, les effets sur la santé sont par nature hypothétiques, difficiles à prévoir, à contrôler ou à évaluer. La modification de l'organisation ne va pas pour autant régler l'ensemble des problèmes de santé tels qu'ils existaient au moment de l'intervention dans les situations de travail. De la même manière, les processus en jeu et leurs effets sur la 
santé ne sont pas toujours réversibles. Par exemple, sur le plan physiologique, certaines lésions peuvent être définitives; du point de vue psychologique et social, la confiance se perd souvent brutalement, et sa construction (ou reconquête) reste toujours longue et incertaine.

Figure 2 : Effets de l'intervention sur l'organisation (effets premiers) et sur la santé (effets seconds).

Figure 2: Effects of the intervention on the organization (first effects) and on the health (second effects)

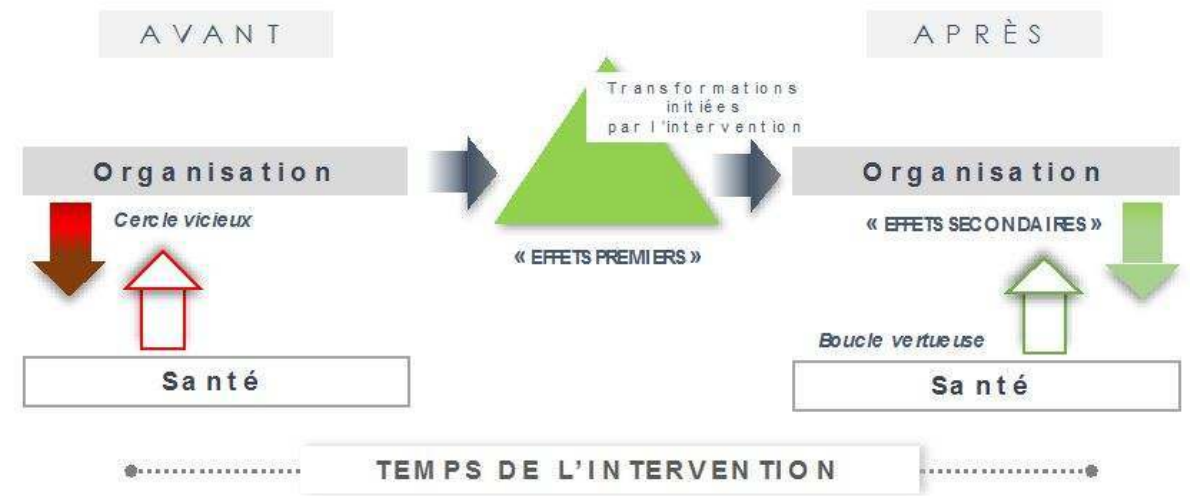

Si nous ne regardons que les indicateurs de "santé » dans une démarche d'évaluation, nous nous privons ainsi de prendre en compte les acquis positifs de la dynamique de l'intervention et son rôle préventif, car les effets premiers visibles sur le fonctionnement de l'organisation ne vont pas pour autant avoir d'impact sur l'ensemble des dégradations de la santé constatées. Seuls les effets réversibles pourront être mis en visibilité, sans que nous puissions en connaître le délai de mise en œuvre.

Dans l'intervention présentée, nous avons été attentifs aux indicateurs d'engagement au travail, au-delà des indicateurs traditionnels de santé-travail, tels que les maladies professionnelles, les accidents du travail, les arrêts longue durée. Ces indicateurs d'engagement tels que les demandes de temps partiels (dont le volume est en augmentation), les arrêts de travail courte durée, les demandes de jours de récupération systématiquement sur les jours les plus pénibles (densité des journées, complexité des enchaînements temporels, pénibilité des activités). L'identification de ces indicateurs comme pertinents et leur analyse ont permis une évaluation première, une forme de pronostics vis-à-vis des indicateurs santé-travail. Car les opérateurs concernés recherchent ainsi une préservation individuelle vis-à-vis de leur environnement professionnel, un évitement, une forme de désengagement.

Si nous nous projetons dans une situation future, l'intervention développe la capacité à ne plus reproduire les mêmes effets que par le passé. L'enjeu devient alors, du point de vue de l'évaluation, de réussir à placer des « capteurs », le plus en amont possible dans le temps, pour pouvoir identifier ce qui bouge en premier.

La santé apparaît être un indicateur incomplet et trop éloigné dans le temps pour pouvoir prédire des améliorations durables. En revanche, les changements de postures, de partis pris et les éléments de discours des parties prenantes apparaissent assez rapidement. Ce sont les premiers « signaux » dans le domaine du visible, consécutifs à des modifications dans les représentations, observables pour attester d'un changement, d'une transformation. 

Traditionnellement, 2 à 3 heures sont allouées à ce type de réunion pour le partager au COPIL. La discussion commence sur des mots, puis sur les sujets de fond présentés qui font l'objet de débats, de discussions actives. Pour une réunion qui devait se terminait à $12 \mathrm{~h}$, elle se termina à $14 \mathrm{~h} 30$ avec la programmation d'une autre journée de travail ensemble pour continuer à débattre sur ce diagnostic. On y voit apparaitre l'effet 1 : une nouvelle lecture partagée de l'existant (ou du passé). Les modalités du dialogue social commencent à bouger.

"stratégique » et décisionnaires, ils se sont sentis concernés. Apparait alors l'effet 4 : " élargissement des catégories d'acteurs "embarqués" ».

Tous ces acteurs réunis avec notre accompagnement se mettent à reconcevoir des pans entiers de l'organisation, en temps réel. Nous identifions alors trois des effets premiers pressentis au début de l'intervention : une nouvelle appréciation de la valeur et du réel (effet 2); l'évolution du périmètre de l'intervention vers les activités managériales et organisatrices du travail (effet 3 ) et, non des moindres, la (co) construction de nouveaux standards \& outils de gestion (effet 5) se substituant aux anciens et se distinguant par une intégration des critères de santé et des critères de performance économique. Les copils ont été très utiles pour obtenir ces effets.

L'évaluation des effets dans ce type de réunion peut se réaliser avec la détection par le ou les consultants, des signaux précurseurs, annonciateurs du changement. Sont-ils objectivables ? Rien n'est sûr. Quand on se dit "ça va changer ", "ça sent bon » et que l'on constate que des personnes ne regardent pas les situations comme avant, cela nous semble annonciateur de mouvements, de changements. Il y a quelque chose de très subjectif de la part des intervenants dans la détection de ces effets premiers et dans la prévision de ce qu'ils peuvent produire dans l'avenir.

\section{Discussion et conclusion}

Nous avons vu, à travers le cadrage théorique et notre exemple illustré, que l'évaluation des interventions sur des problématiques complexes de santé au travail, telles que la prévention des TMS et des RPS, est un enjeu prépondérant aussi bien pour les intervenants eux-mêmes que pour les institutions de prévention et les universitaires. Du point de vue des consultants, des questionnements persistent toujours : que produisentils avec leurs interventions? Quels impacts et quels effets engendrent-ils sur les organisations?

51 Nos résultats alimentent l'état de l'art en proposant une caractérisation des «effets premiers ", premiers indicateurs de transformation par l'intervention, selon cinq types de signaux que l'intervenant rencontre régulièrement: nouvelle lecture partagée de l'existant, nouvelle appréciation de la valeur, évolution du périmètre de l'intervention vers les activités managériales et organisatrices du travail, élargissement des catégories d'acteurs "embarqués ", et, construction de nouveaux standards et outils de gestion. Cependant, l'identification de ces effets premiers est discutable en termes d'exhaustivité, de valeur prédictive et d'usage dans l'intervention.

52 Tout d'abord, bien que la typologie, ici appuyée par notre exemple dans le secteur des transports, se vérifie dans nombre des interventions réalisées dans notre Cabinet Conseil, 
il semble prudent de nuancer nos résultats: les cinq signaux identifiés sont-ils généralisables pour l'ensemble des interventions ? Existe-t-il d'autres signaux ? Et est-il possible de les catégoriser de manière différente? Pour analyser la genèse des phénomènes de transformation, leurs relations de causes à effets et leur visibilité, nous avons également fait le choix de hiérarchiser l'apparition des signaux précurseurs et annonciateurs d'un changement. Mais est-il possible de hiérarchiser différemment ces signaux? Ainsi en poursuivant notre capitalisation des interventions au sein du Cabinet Conseil, il sera possible de conforter, ou à défaut d'ajuster cette caractérisation.

Par ailleurs, nos résultats montrent que les effets premiers sont souvent précurseurs d'effets seconds, dont la mise en visibilité nécessite un temps plus long que celui de l'intervention, notamment ceux impactant la santé. En effet, l'état de santé étant fluctuant, ses dégradations ou améliorations sont plus ou moins visibles et palpables (Volkoff \& Molinié, 2011). Comment donc parvenir à évaluer l'efficacité des interventions de prévention de la santé? Comment évaluer les effets de l'intervention sur des phénomènes « au long court » qui mettront pour certains d'entre eux plusieurs années à parvenir dans le spectre du visible ? Nous maintenons l'hypothèse que les effets premiers, plus facilement identifiables sur le temps de l'intervention, ont une valeur prédictive sur les effets seconds. Ce caractère prédictif, en termes d'effets sur la santé, mais également sur la performance, reste à investiguer plus particulièrement pour prolonger nos travaux: les effets premiers sont-ils annonciateurs de changement? Le travail d'organisation (Clot, 2002) se prolonge au-delà de l'intervention par des modifications et des transformations des organisations de travail, mais ces modifications vont-elles se traduire, à un moment donné dans l'histoire des organisations, par des effets seconds ? Si oui, comment les mesurer? Et comment les suivre lorsque l'intervenant a quitté l'organisation après la fin de l'intervention? Autant de questions qui sont encore à étudier au niveau méthodologique et pragmatique.

Enfin, notre caractérisation des effets premiers a posteriori de l'intervention pose la question de leur usage dans l'intervention: comment reconnaitre puis embarquer ces signaux en cours d'intervention? Il s'agirait ici, en développant un dispositif d'intervention, de compléter l'évaluation ouverte que peuvent réaliser les intervenants, notamment par leur pratique réflexive (INRS \& ANACT, 2017).

\section{BIBLIOGRAPHIE}

Arnoud, J., \& Falzon, P. (2017). Intervenir « sur » l'organisation : vers un cadre méthodologique. In A.-L. Ulmann, A. Weill-Fassina, \& T.H. Benchekroun (Eds.), Intervenir, Histoires, recherches, pratiques (pp. 155-163), Toulouse : Octarès.

Avargues, M.-C., Champagne, F., Contandriopoulos, A.-P., \& Denis, J.-L. (2000). L'évaluation dans le domaine de la santé : concepts et méthodes. Revue d'épidémiologie et de santé publique, 48(6), 517-539.

Clot, Y. (2002). Clinique de l'activité et répétition. Clinique Méditerranéenne, 66, 31-54. 
Daniellou, F. (2006). La place de l'évaluation dans la pratique de l'ergonome. Actes des Journées de Bordeaux sur la pratique de l'ergonomie.

Heddad, N., \& Biquand, S. (2015). La pluridisciplinarité en pratique - Retour d'expérience et questionnement disciplinaire. In F. Hubault (Ed.), Actes du Séminaire Paris 1 La dynamique des métiers de l'ergonomie (pp. 55-60). Toulouse : Octarès.

Hendrick, H. W. (2003). Determining the cost-benefits of ergonomics projects and factors that lead to their success. Applied Ergonomics, 34(5), 419-427.

INRS \& ANACT (2017). Évaluer les interventions de prévention RPS - TMS. Guide à l'usage des intervenants. Toulouse : Octarès. $56 \mathrm{p}$.

Landry, A. (2008). L'évaluation de l'intervention ergonomique : de la recherche évaluative à la proposition d'outils pour la pratique. Thèse de doctorat en ergonomie, Université Bordeaux 2.

Pagnac, L., \& Arnaud, S. (2002). De l'ergonomie parapluie à l'ergonome qui se mouille. Actes des journées de Bordeaux sur la pratique de l'ergonomie. «Ergonome interne, ergonome externe : les formes de l'engagement".

Schön, D.A. (1994). Le praticien réflexif. À la recherche du savoir caché dans l'agir professionnel. Montréal : Éditions Logiques.

Volkoff, S., \& Molinié, A. F. (2011). L'écheveau des liens santé-travail, et le fil de l'âge. In A. Degenne, C. Marry, \& S. Moulin (Eds.), Les catégories sociales et leurs frontières (pp. 323-344). Laval, Québec Presses de l'Université de Laval.

\section{RÉSUMÉS}

Depuis de nombreuses années, la question de l'évaluation de l'intervention sur les Troubles Musculo squelettiques (TMS) et les Risques Psychosociaux (RPS) est une préoccupation pour tous les acteurs. Confronté à ces sujets complexes, l'intervenant est souvent mis en difficulté pour repérer les indicateurs de la pertinence du dispositif qu'il mobilise. La capitalisation des interventions menées au sein de notre Cabinet Conseil depuis 25 ans nous a permis d'identifier cinq signaux à détecter, précurseurs d'«effets premiers». À partir de l'exemple d'une intervention menée dans le secteur des transports, nous proposons de les caractériser: une nouvelle lecture partagée de l'existant, une nouvelle appréciation de la valeur, une évolution du périmètre de l'intervention vers les activités managériales et organisatrices du travail, un élargissement des catégories d'acteurs "embarqués", et, une construction de nouveaux standards et outils de gestion qui se distinguent par une intégration des critères de santé et de performance économique.

The question of the evaluation of the intervention on Musculoskeletal Disorders (MSD) and Psychosocial Risks (PSR) is a concern for all the actors for many years. The consultant is confronted with these complex subjects and he is often got into real trouble to identify the indicators of the sucess of the strategy he mobilized. The capitalization of interventions in our consulting firm for 25 years allowed us to identify five signals, the "first effects". We suggest characterizing it from the example of an intervention in the sector of transport: a new sharing reading of the existing, a new appreciation of the value, an evolution of the area of the intervention to the managerial and organizing activities at work, an extension of the categories of actors "on board", and a construction of new standards and management tools which integrate health and economic performance indicators. 
INDEX

Mots-clés : organisation du travail et systèmes sociotechniques, état de santé, performance du système et évaluation, entretiens

Keywords : work organization and sociotechnical systems, state of health, system performance and evaluation, interviews

\section{AUTEURS}

\section{SANDRINE NAHON}

Solutions Productives, PA de Ker Lann, 35070 Bruz - ergonomie@solutions-productives.com CÉLIA QUERIAUD

Solutions Productives, PA de Ker Lann, 35070 Bruz - ergonomie@solutions-productives.com CÉCILE MEGE PINEY

Solutions Productives, PA de Ker Lann, 35070 Bruz - ergonomie@solutions-productives.com ISABELLE THIBAULT

Solutions Productives, PA de Ker Lann, 35070 Bruz - ergonomie@solutionsproductives.com 